\title{
Conditionally Controlling Human TLR2 Activity via Trans- Cyclooctene Caged Ligands
}

\author{
Michel J. van de Graaff," Timo Oosenbrug," Mikkel H. S. Marqvorsen, Clarissa R. Nascimento, \\ Mark A. R. de Geus, Bénédicte Manoury, Maaike E. Ressing,* and Sander I. van Kasteren*
}

Cite This: Bioconjugate Chem. 2020, 31, 1685-1692

Read Online

\section{ACCESS \\ Џlll Metrics \& More \\ Article Recommendations \\ Supporting Information}

ABSTRACT: Toll-like receptors (TLRs) are key pathogen sensors of the immune system. Their activation results in the production of cytokines, chemokines, and costimulatory molecules that are crucial for innate and adaptive immune responses. In recent years, specific (sub)cellular location and timing of TLR activation have emerged as parameters for defining the signaling outcome and magnitude. To study the subtlety of this signaling, we here report a new molecular tool to control the activation of TLR2 via "click-to-release"-chemistry. We conjugated a bioorthogonal trans-cyclooctene (TCO) protecting group via solid support to a critical position within a synthetic TLR2/6 ligand to render the compound unable to initiate signaling. The TCO-group

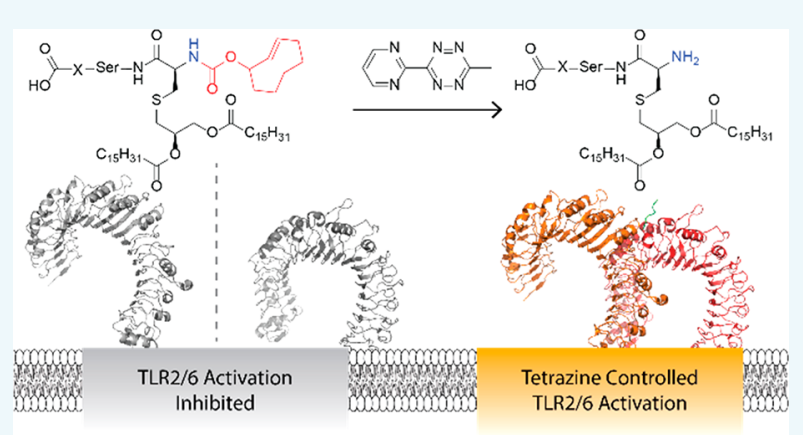
could then be conditionally removed upon addition of a tetrazine, resulting in restored agonist activity and TLR2 activation. This approach was validated on RAW264.7 macrophages and various murine primary immune cells as well as human cell line systems, demonstrating that TCO-caging constitutes a versatile approach for generating chemically controllable TLR2 agonists.

$\mathrm{T}$ oll-like receptors (TLRs) play a pivotal role in the (innate) immune system. Upon recognition of conserved microbial structures, TLRs dimerize and induce signaling events that culminate in the production of cytokines, chemokines, and costimulatory molecules. ${ }^{1}$ These serve both to directly combat infection and to attract and activate other cells of the innate and adaptive immune system. ${ }^{2}$ For this reason, synthetic TLR agonists are potent therapeutic compounds for preventing/treating infections ${ }^{3,4}$ but also for boosting anticancer immune responses. ${ }^{5}$ Excessive TLRinduced inflammation can, however, have deleterious effects. Infection-associated sepsis ${ }^{6}$ and inflammatory and autoimmune diseases, such as asthma ${ }^{7}$ and systemic lupus erythematosus (SLE), ${ }^{8}$ are marked by aberrant TLR-signaling. Thus, a detailed understanding of the processes that shape the outcome of TLR signaling is required for the rational design of novel therapeutics that elicit beneficial effects while preventing immune pathogenesis.

Over the past decade, it has become apparent that TLRs display complex, dynamic behavior within (immune) cells. ${ }^{9}$ The specific (sub)cellular locations where these receptors can be activated are tightly controlled. For example, the intracellular TLRs 3, 7, 8, and 9 are produced as inactive precursors that are processed locally in endolysosomal vesicles by $\mathrm{pH}$ sensitive proteases. This ensures they can only engage ligands at this specific location. ${ }^{10-13}$ Additionally, different signaling outcomes may be realized from distinct cellular sites, as exemplified by TLR4: when ligated at the cell surface, TLR4 assembles a signaling platform via the adaptor protein MyD88 to induce the activation of the transcription factors nuclear factor $-\kappa \mathrm{B}(\mathrm{NF}-\kappa \mathrm{B})$ and activator protein- 1 (AP- 1$).{ }^{14}$ This results in the expression of pro-inflammatory cytokines and chemokines. When, on the other hand, for the same receptor signals from an endosome, ${ }^{15}$ it uses the adaptor protein TRIF to induce the activation of interferon regulatory factors (IRFs), ${ }^{16}$ which in turn initiates the production of antiviral type I interferons (IFN-I). For the cell-surface resident TLR2, location-specific signaling outcomes have also been reported, ${ }^{17-19}$ although the signaling dynamics and their relation to immune activation appear less well understood.

To study this complexity of signaling properly, specialized chemical tools have been developed that allow controlled induction of TLR activation. For example, biotinylated TLRligands immobilized on surfaces have been used to spatially restrict signaling of the receptor to the cell surface. ${ }^{20}$ Photolabile protecting groups introduced to key residues on TLR-agonists have been used to control the signaling of the receptor in time. Upon UV-irradiation, the protecting group

Received: April 19, 2020

Revised: May 27, 2020

Published: June 8, 2020 
can be removed to initiate TLR binding and signaling. ${ }^{21-25}$ Potential drawbacks of this photochemistry based approach, include the induction of phototoxicity as well as the limited in vivo tissue penetrance of UV-light.

Bioorthogonal protection/deprotection strategies can offer a solution here. By using protecting groups that can be selectively removed with nontoxic chemical reagents, the above limitations can be circumvented. For such a reaction to be of use, it has to be fast, nontoxic, and synthetic access to the reagents must be feasible. ${ }^{26,27}$ One reaction that meets these requirements is the inverse electron-demand Diels-Alder (IEDDA)/pyridazine elimination tandem reaction between a 2-substituted trans-cyclooctene (2-TCO) and a tetrazine, a socalled "click-to-release"-reaction (C2R). ${ }^{28}$ Reactivity of TCO toward tetrazines originates from the high degree of ring strain induced by the trans-configuration of the olefin. ${ }^{29}$ After a $[4+$ 2] cycloaddition, 4,5-dihydropyridazine is formed while expelling nitrogen in the process. ${ }^{28}$ This is followed by a tautomerization to liberate the carbamate functionality, which quickly decarboxylates to release a free amine functional group and carbon dioxide (Figure 1). The above C2R-reaction has

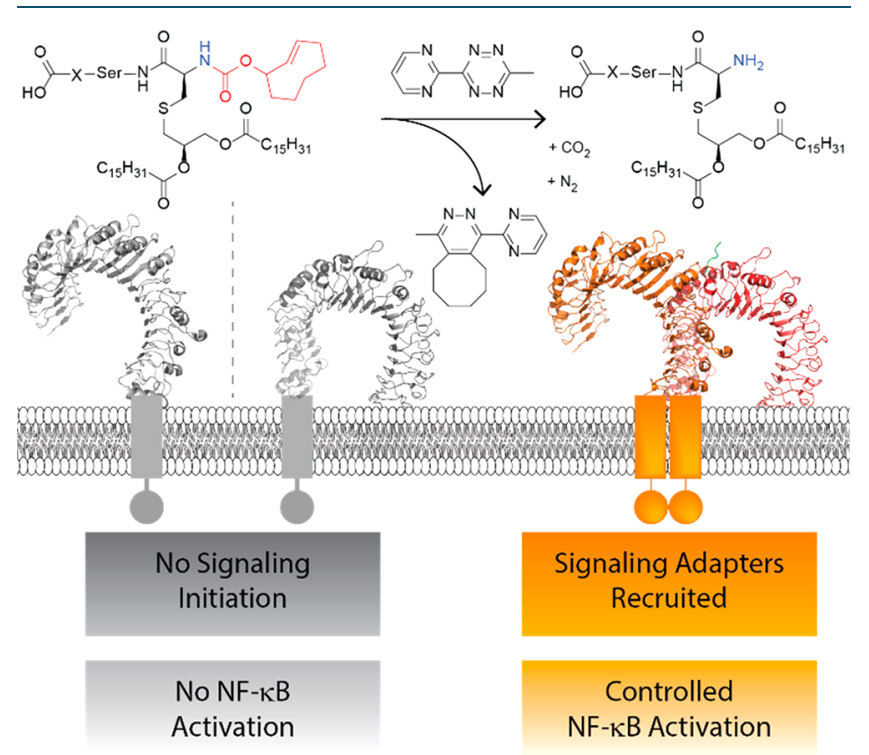

Figure 1. Schematic of conditionally inducing TLR2 activation via "click-to-release" chemistry. Signaling via TLR2/6 by an agonist protected with trans-cyclooctene (TCO, indicated in red) is selectively induced after a tetrazine is applied to remove the caging moiety. Figure is based on the crystal structure of TLR2 $/ 6 .{ }^{39} \mathrm{X}=\mathrm{Lys}_{4}$ or TEG.

gained popularity due to its rapid ligation and elimination kinetics $^{30-32}$ and has found use in antibody-drug conjugates, ${ }^{33,34}$ protein activation, ${ }^{35,36}$ RNA synthesis, ${ }^{37}$ and ondemand T-cell activation. ${ }^{38}$ Furthermore, tetrazine doses required for inducing this reaction are nontoxic, thus, permitting studies even in live animals. ${ }^{33,34,36,38}$ The speed of the reaction drives TCO-elimination in an almost instantaneous "switch-like" manner, which is of crucial importance when investigating the complex kinetics of receptor signaling. ${ }^{31,32}$

Here, we set out to develop a C2R-approach for conditionally controlling the activation of TLRs. We focused on TLR2 (Figure 1), because many of its downstream signaling properties remain to be elucidated. While most TLRs form homodimers upon ligation, TLR2 forms heterodimer complexes with TLR1 or TLR6, each having distinct ligand-binding specificities. ${ }^{40}$ We conjugated TCO to a known TLR2/6 agonist in such a way that it obstructs receptor heterodimerization, so that only after elimination of the protecting group by a tetrazine, receptor dimerization can take place, leading to the induction of inflammatory signaling (Figure 1). We investigated the use of this approach for studying the kinetics of TLR2/6 activation as well as the cytokine profile after activation. The low toxicity of this approach allows the study of these processes in model cell lines but also in primary immune cells.

\section{RESULTS AND DISCUSSION}

One of the best-defined synthetic TLR2/6 agonists is $\mathrm{Pam}_{2} \mathrm{CSK}_{4}\left(\mathrm{P}_{2} \mathrm{~K}_{4}\right)$, for which both a crystal structure of the ligand bound to murine TLR2/TLR $6^{39}$ and extensive structure-activity relationship (SAR) data $^{41}$ are available. The two palmitoyl tails of $\mathrm{P}_{2} \mathrm{~K}_{4}$ strongly bind in a hydrophobic pocket of TLR2, whereas the Cys-Ser amide bond engages in a hydrogen bond with TLR6.

Substitution of the N-terminus diminishes ligand activity, likely through disrupting TLR2/6 dimerization, while maintaining strong binding to TLR2. ${ }^{23}$ The amine-residue critical to this interaction is therefore an ideal conjugation site for a bioorthogonal protecting group.

Synthesis of the Unmodified and TCO-Caged TLR2/6 Ligand $\mathbf{P}_{2} \mathbf{K}_{4}$. To explore inverse electron-demand DielsAlder (IEDDA)/pyridazine (de)protection as an approach for the guided activation of TLR2, we synthesized both a TCOcaged derivative of $\mathrm{P}_{2} \mathrm{~K}_{4}(4$, Scheme 1$)$ as well as an uncapped

\section{Scheme 1. Synthesis of TCO-Protected $\mathrm{Pam}_{2} \mathrm{CSK}_{4}-$} Analogue ${ }^{a}$

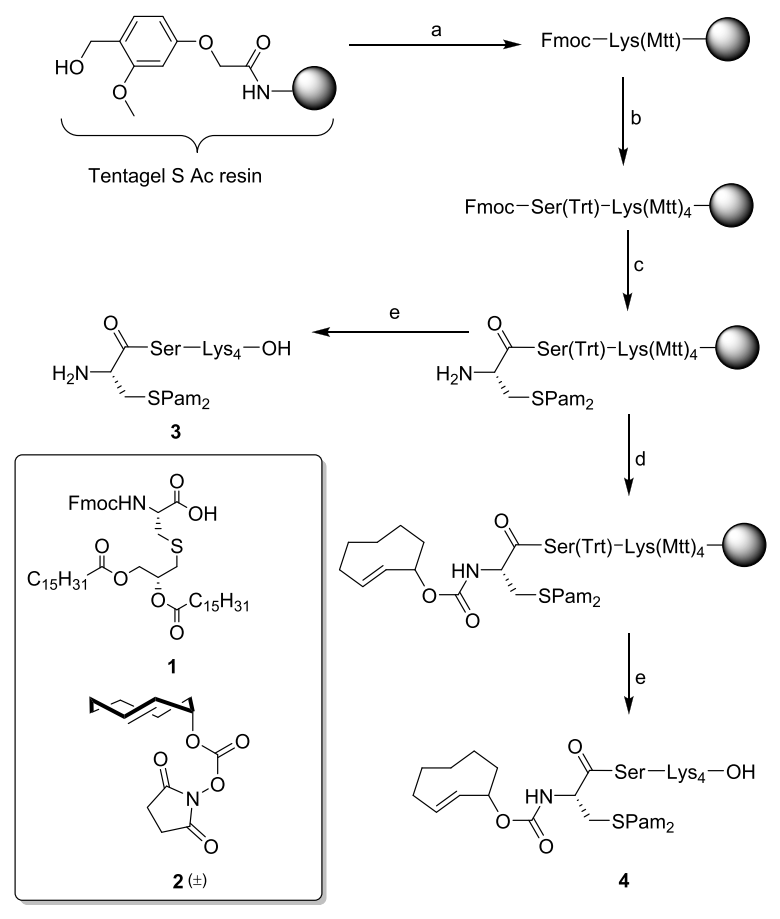

a (a) Fmoc-Lys(Mtt)-OH, DIC, DMAP, rt; (b) HCTU-mediated SPPS, rt; (c) (1) $20 \%$ piperidine, DMF, rt; (2) Compound 1, HCTU, DiPEA, rt; (3) $20 \%$ piperidine in DMF, rt; (d) Compound 2, DiPEA, rt; (e) $5 \%$ TFA, $1 \%$ TIS, $1 \% \mathrm{H}_{2} \mathrm{O}$ in DCM, rt. 
control (3, Scheme 1). Synthesis was performed on the Tentagel S AC resin. The serine side chain was protected as a trityl (Trt) ether, while lysine side chains were protected with 4-methyltrityl (Mtt) to enable the full deprotection and resincleavage of products under dilute TFA conditions. This was necessary due to TCO's propensity to isomerize under acidic conditions. ${ }^{42}$ Pure building block $\mathbf{1}$, with the central carbon in the diacylglycerol group R-configured, was used as the activity of the S-isomer is significantly lower. ${ }^{43}$ The TCO-caged ligand was obtained after reaction with 2 . Compounds 3 and 4 were then cleaved from the solid support using a mixture of 5\% TFA in DCM, thereby minimizing TCO isomerization, followed by HPLC purification.

Activity of the TCO-Caged TLR2 Ligand Is Suppressed and Is Restored upon Applying a Chemical Deprotection Agent. To assess whether protection of the critical amine in $\mathrm{P}_{2} \mathrm{~K}_{4}$ as a TCO-carbamate rendered the compound unable to activate TLR2/6-signaling, the murine macrophage cell line RAW264.7 was treated with 4 (Figure 2A, control) and as a measure of TLR2 activation, nuclear translocation of the transcription factor NF- $\kappa \mathrm{B}$ was visualized using confocal microscopy. Cells treated with 4 exhibited no nuclear translocation of NF- $\kappa \mathrm{B}$, indicating that the TCO-cage prevents TLR2 activation (Figure 2B). Next, we determined whether removal of the protecting group could restore agonist activity. Upon addition of $10 \mu \mathrm{M}$ 3-methyl-6-pyrimidyltetrazine $(\mathrm{Tz})^{44}$ to cells pretreated with 4 (Figure $2 \mathrm{~A},+\mathrm{Tz}$ ), nuclear NF- $\kappa \mathrm{B}$ was detected after $30 \mathrm{~min}$ (Figure $2 \mathrm{~B}$ ), indicating that $\mathrm{P}_{2} \mathrm{~K}_{4}$ was liberated by the added $\mathrm{Tz}$ to induce TLR2 signaling.

To further evaluate the effect of TCO-deprotection, downstream transcriptional activity of NF- $\kappa \mathrm{B}$ was assessed using the commercially available RAW-Blue reporter cell line. These cells produce secreted embryonic alkaline phosphatase (SEAP) expressed from an NF- $\kappa \mathrm{B}$ inducible promoter. Cells were treated with the TCO-caged compound 4 as above or with control compound 3. After $24 \mathrm{~h}$, SEAP levels were determined in the culture supernatant using a colorimetric assay. Cells treated with $0.01-1 \mathrm{nM} 4$ displayed marginal levels of NF- $\kappa \mathrm{B}$ activity compared to cells stimulated with the free ligand 3 (Figure 2C, dark green bars compared to blue line). Only at a high ligand dose $(10 \mathrm{nM})$, the caged ligand did induce some residual activity. The chemical trigger $\mathrm{Tz}$ restored compound 4-induced NF- $\kappa \mathrm{B}$-SEAP levels to those of cells treated with 3 (Figure 2C, bright green bars compared to blue line), whereas NF- $\kappa$ B-SEAP was not induced when treating cells with $\mathrm{Tz}$ alone. These data confirm that $\mathrm{Tz}$-induced ligand uncaging restores the agonist activity of $\mathbf{4}$ and that this translates to TLR2 activation and a cellular response.

Next, we assessed whether conditionally controlled TLR2 activation resulted in the production of downstream inflammatory mediators in primary bone marrow derived macrophages (BMDMs) and bone marrow derived dendritic cells (BMDCs) (Figure 2D,E and 3A). When treated with 4, both types of innate immune cells selectively secreted the NF$\kappa \mathrm{B}$-induced cytokine tumor necrosis factor- $\alpha(\mathrm{TNF} \alpha)$ when the agonist was liberated using $\mathrm{Tz}$ (Figure $2 \mathrm{D}$ and $3 \mathrm{~A}$ ). Additionally, compound 4-treated BMDMs selectively upregulated the macrophage activation marker F4/80 after uncaging (Figure 2E). To exclude the aspecific induction of inflammatory mediators, for instance due to cellular stress, we confirmed that 4 did not induce $\mathrm{TNF} \alpha$ production under uncaging conditions in TLR2 knockout ( $\triangle T$ TLR2) BMDMs and

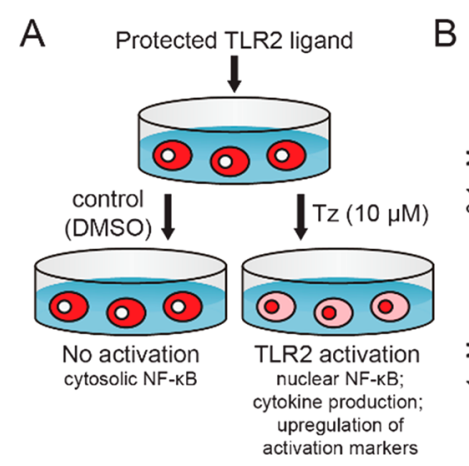

C

C RAW-Blue macrophages
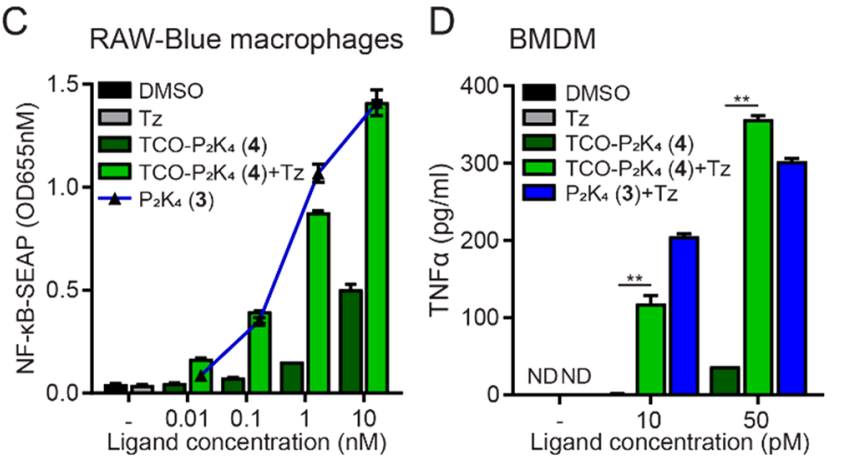

E

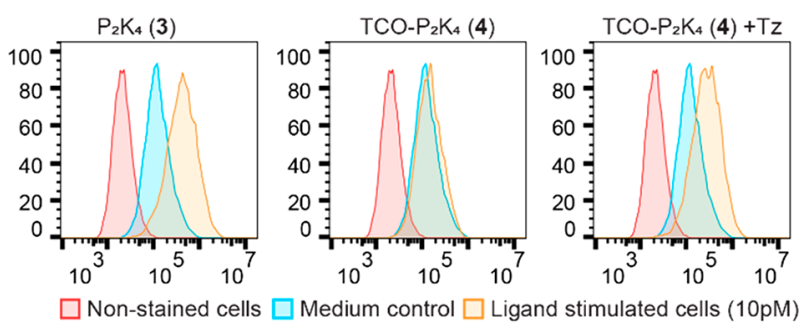

Figure 2. Controlling the activity of murine TLR2 with TCO-caged $\mathrm{P}_{2} \mathrm{~K}_{4}$ on macrophages: (A) experimental outline for conditionally controlling TLR2 activity. After a 45-60 min preincubation of cells with the caged ligand, medium is replaced with tetrazine $(\mathrm{Tz})$ containing medium. This triggers rapid removal of the protecting group, thereby restoring agonist activity and resulting in TLR2induced NF- $\kappa$ B activation. (B) RAW-Blue macrophages were fixed after $30 \mathrm{~min}$ and stained for the NF- $\kappa \mathrm{B}$ subunit p65 (in red). (C) Levels of NF- $\kappa$ B-induced SEAP in the culture supernatant of RAWBlue reporter cells $24 \mathrm{~h}$ after $\mathrm{Tz}$ treatment. (D) Conditionally induced TNF $\alpha$ by BMDMs as surveyed by ELISA of culture supernatant $6 \mathrm{~h}$ after Tz treatment. ND not detected; NS, not significant; **, $p<0.01$ (Students $t$ test). Purity checks of the cell cultures used for are provided in Figure S1. Data are representative of one (B and C), two (E), or four (D) independent experiments.

BMDCs, even at high ligand doses (Figure S2). For CD11 ${ }^{+}$ splenic DCs that were directly treated ex vivo, conditionally controlled $\operatorname{TNF} \alpha$, and interleukin-6 (IL-6) production was observed (Figure 3B,C), demonstrating the robustness of the TCO-(un)caging strategy for use in murine cell lines, differentiated immune cells, and primary cells upon treatment with 4 and Tz.

These combined findings illustrate that TCO is a suitable cage for inhibiting mouse TLR2/6 ligand activity and that tetrazine-induced uncaging sufficiently recovers agonist activity to induce TLR2 signaling. This confirms the potential of the IEDDA/pyridazine elimination tandem reaction to conditionally control TLR2 activation. 


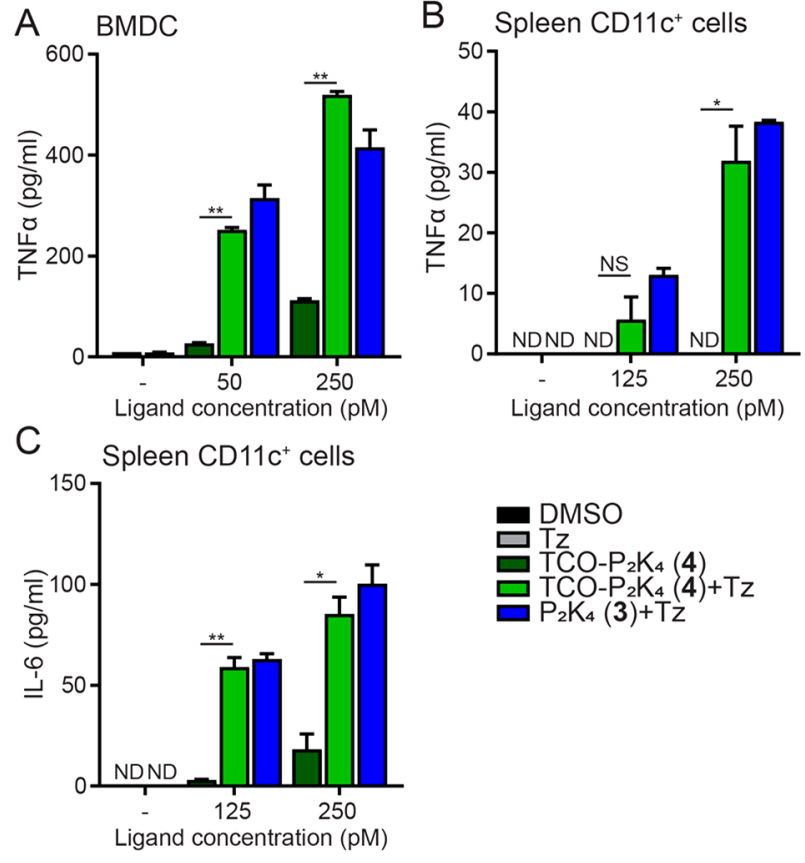

Figure 3. Controlling the activity of murine TLR2 with TCO-caged $\mathrm{P}_{2} \mathrm{~K}_{4}$ on dendritic cells: $(\mathrm{A}-\mathrm{C})$ Dendritic cells were treated as described in Figure 2A, and conditionally induced TNF $\alpha$ or IL6 production was surveyed by ELISA in culture supernatant $6 \mathrm{~h}(\mathrm{~A})$ or $16 \mathrm{~h}(\mathrm{~B}, \mathrm{C})$ after Tz treatment. ND not detected; NS, not significant; *, $p<0.05$; **, $p<0.01$ (Students $t$ test). Because BMDCs and CD $11 \mathrm{c}^{+}$splenic DCs responded to the free, noncapped compound 3 with different sensitivities compared to BMDMs, different (indicated) doses were used to yield robust TLR2 responses. Purity checks of the cell cultures used for are provided in Figure S1. Data are representative of four (A) or two (B and C) independent experiments.

Substantial Residual Activity of the TCO-Caged TLR2 Ligand Limits Its Use in Human Cells. Recently, a number of striking differences have been reported in the molecular mechanisms employed by mouse and human TLR2 to transduce signals ${ }^{18}$ (manuscript under review). To study the complexity of human TLR2 signaling it is, therefore, important that chemical tools are also applicable in human cells. We therefore evaluated the conditional induction of human TLR2 activity with $\mathbf{4}$ next.

We treated human TLR2-expressing melanoma cell line MelJuso as above and visualized NF- $\kappa \mathrm{B}$ localization. Nuclear NF- $\kappa \mathrm{B}$ levels were clearly enriched after $10 \mu \mathrm{M} \mathrm{Tz}$ treatment for all ligand doses tested (Figure 4A), demonstrating that, also in human cells, uncaging of 4 results in enhanced TLR2 activity. We, however, detected considerable amounts of nuclear NF- $\kappa$ B in cells treated only with the caged compound. To exclude TLR2 overexpression related or cell type-specific artifacts, we next employed the human monocyte reporter cell line THP1-Dual (Invivogen), which expresses NF- $\kappa$ B-inducible SEAP, analogous to the above mouse reporter macrophages. TLR2-induced NF- $\kappa$ B activity was confirmed for 3 and Tztreatment restored SEAP induction by THP1-Dual cells treated with 4 to the levels induced by 3 (Figure 4B). Yet, also in these immune cells, we observed marked residual activity of the caged compound for doses $\geq 0.1 \mathrm{nM}$.

The combined data suggest that, while Tz-induced uncaging of 4 conditionally induces human TLR2 activity, its experimental use in human cells is limited due to residual

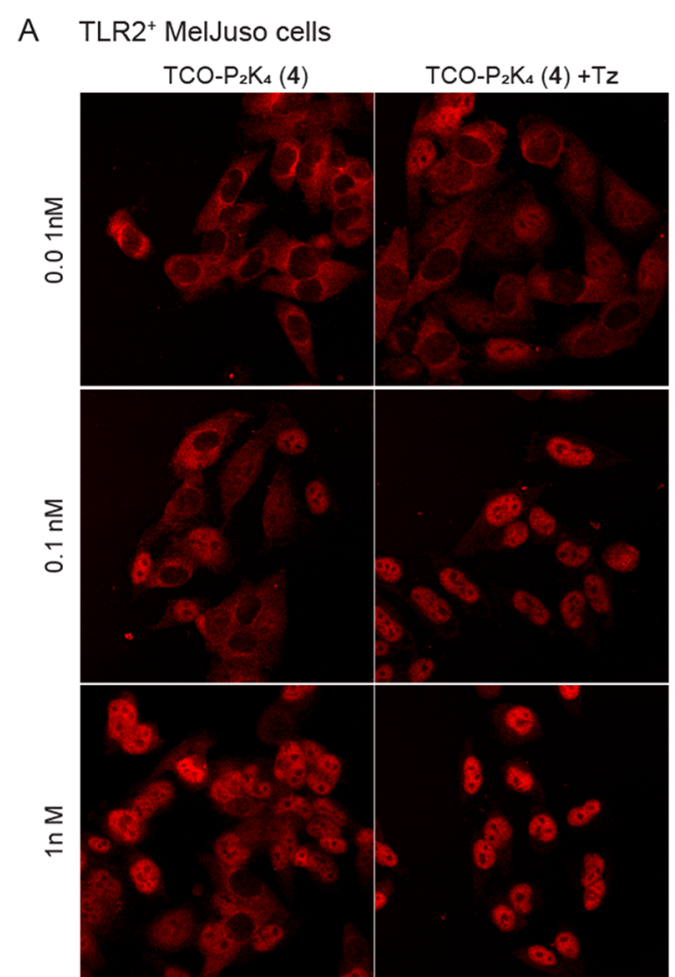

B THP1-Dual monocytes

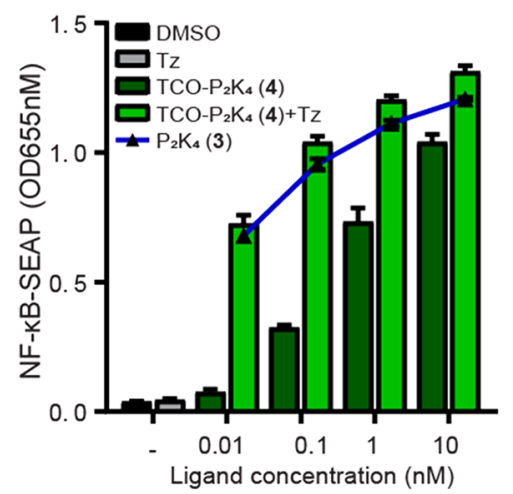

Figure 4. Substantial residual activity induced by TCO-caged $\mathrm{P}_{2} \mathrm{~K}_{4}$ hampers experiments with human cells. (A) MelJuso TLR2YFP cells were fixed $30 \mathrm{~min}$ after ligand uncaging was induced and stained for the NF- $\kappa$ B subunit p65 (in red). (B) NF- $\kappa$ B-SEAP levels in the culture supernatant of THP1-Dual monocytes $24 \mathrm{~h}$ after conditionally inducing TLR2 activation. Data are representative of two (A) or three (B) independent experiments and cells were treated as described in Figure 2A.

activity exerted by the protected compound. Only at low doses $(\leq 0.01 \mathrm{nM})$ did compound 4 not induce substantial TLR2 activation. However, at these doses, NF- $\kappa \mathrm{B}$ is suboptimally activated after ligand deprotection, evidenced by a limited number of cells showing modest levels of NF- $\kappa \mathrm{B}$ nuclear translocation (Figure 4A) and low NF- $\kappa$ B-reporter levels (Figure 4B). We hypothesized that the discrepancy between our mouse and human TLR2 data (Figures 2-4) resulted from structural differences at TLR2 sites important for ligand binding, ${ }^{45}$ leading to altered ligand specificities and affinities. ${ }^{46}$ $\mathrm{We}$, therefore, next attempted to redesign the TCO-caged TLR2 ligand so that it would robustly induce NF- $\kappa$ B activation 
in human cells with a better signal-to-noise ratio over a broad concentration range.

Design and Synthesis of a Human-TLR2/6-Compatible Caged Ligand. We opted to increase the steric bulk of the TCO-cage, to assess whether this could better prevent receptor dimerization in human cells. For this, we used a bifunctional TCO-group (6, Scheme 2), ${ }^{33}$ enabling the

Scheme 2. Synthesis of Human TLR2/6 Compatible Caged Ligands ${ }^{a}$
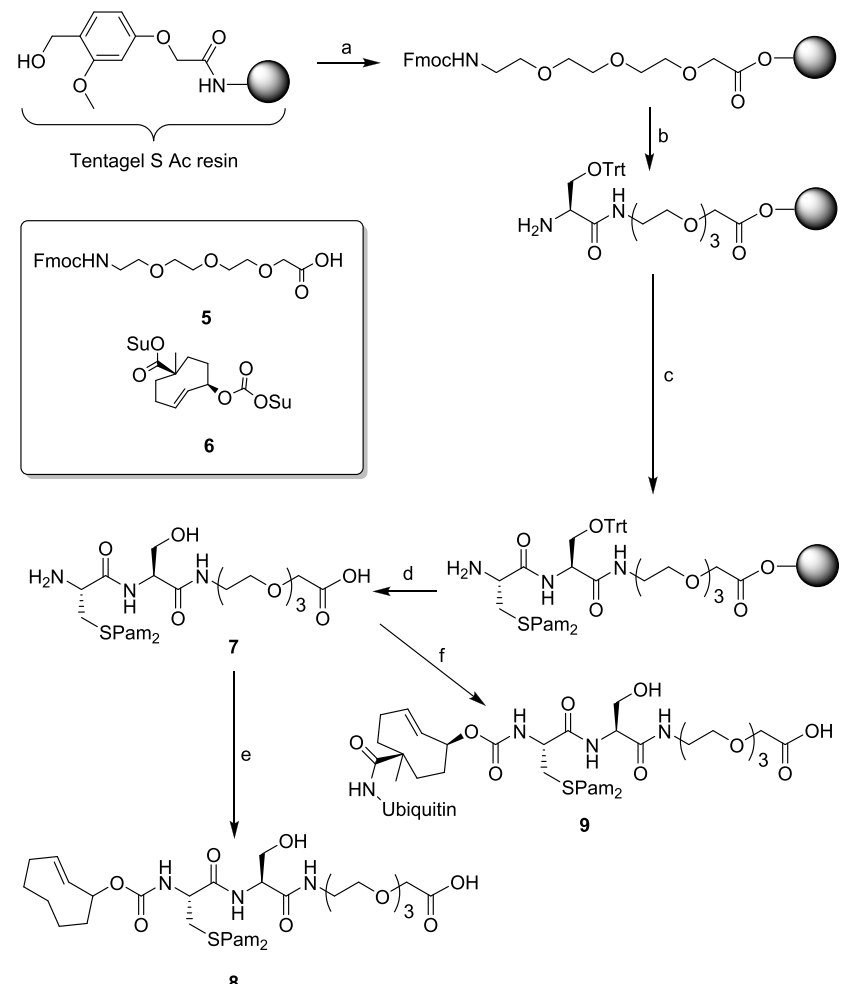

${ }^{a}$ (a) Compound 5, DIC, DMAP, rt; (b) (1) 20\% piperidine in DMF, rt; (2) Fmoc-Ser(OTrt)-OH, HCTU, DiPEA, rt; (3) $20 \%$ piperidine in DMF, rt; (c) (1) compound 1, HCTU, DiPEA, rt; (2) $20 \%$ piperidine in DMF, rt; (d) 20\% TFA, $2.5 \%$ TIS, $2.5 \% \mathrm{H}_{2} \mathrm{O}$ in DCM, rt; (e) compound 2, DiPEA, rt; (f) (1) compound 6, DiPEA, rt; (2) ubiquitin, DiPEA in DMSO, rt.

introduction of a bulky hydrophilic substituent at the second modification site. The small protein ubiquitin (Ub) was conjugated to this position, in view of its favorable properties: it is an $8.6 \mathrm{kDa}$ water-soluble, accessible, and thermally stable protein. Conjugating such a large molecule to an activated ester on solid phase often suffers from extremely low coupling efficiencies. Hence, we opted for a solution-phase coupling strategy.

Deprotection of the lysine residues of $\mathrm{P}_{2} \mathrm{~K}_{4}$ after protein conjugation in a solution-phase strategy, while abstaining from using acid- or base-labile protecting groups, quickly leads to complex synthetic routes. In contrast to the lipidated CysSer dipeptide motif, the C-terminal lysine residues of the agonist are not crucial for inducing TLR2 activation ${ }^{39}$ and substitution of the $\mathrm{K}_{4}$-tail with ethylene glycols has previously been shown to be tolerated. ${ }^{47} \mathrm{We}$, therefore, chose to replace the $\mathrm{K}_{4}$-tail of $\mathrm{P}_{2} \mathrm{~K}_{4}$ with triethylene glycol (TEG), to facilitate the synthesis of a Ub-TCO-caged TLR2/6 ligand (compound 9, Scheme 2). For comparison, we also synthesized the free, noncapped (7) and TCO-caged (8) Pam $_{2}$ CSTEG ( $\mathrm{P}_{2}$ TEG) equivalents. The ability of compound 8 to react with tetrazine was confirmed in an LC-MS experiment (Figure S8).

Analogous to the synthesis of compound 3, compound 7 was synthesized on resin for ease of purification of the intermediates. After cleavage from the resin, 7 was purified using RP-HPLC. Compound 7 was then treated with either TCO-OSu (2) or bis-functionalized TCO (6) in solution phase. After LC-MS indicated complete consumption of compound 7, the mixture was added to a solution of ubiquitin in DMSO. After having stirred the reaction for 7 days, HRMS indicated the formation of a ubiquitin conjugate containing one, two, or three ligands. Interestingly, HPLC purification of compound 9 led to the isolation of two separate fractions both containing a compound with the molecular mass of a singly conjugated product. Since ubiquitin contains seven lysine residues as well as a free $\mathrm{N}$-terminus, different isoforms can be expected to form. Both fractions were tested in biological assays and are referred to as fraction $\mathrm{A}$ or fraction $\mathrm{B}$ in the Supporting Information.

TCO-Caged $\mathrm{P}_{2}$ TEG Shows Enhanced Performance in Human Cells. The $\mathrm{P}_{2}$ TEG-derived constructs (7-9) were tested in the THP1-Dual reporter cell system along with 4 (Figure 5). Both collected fractions of compound 9 gave

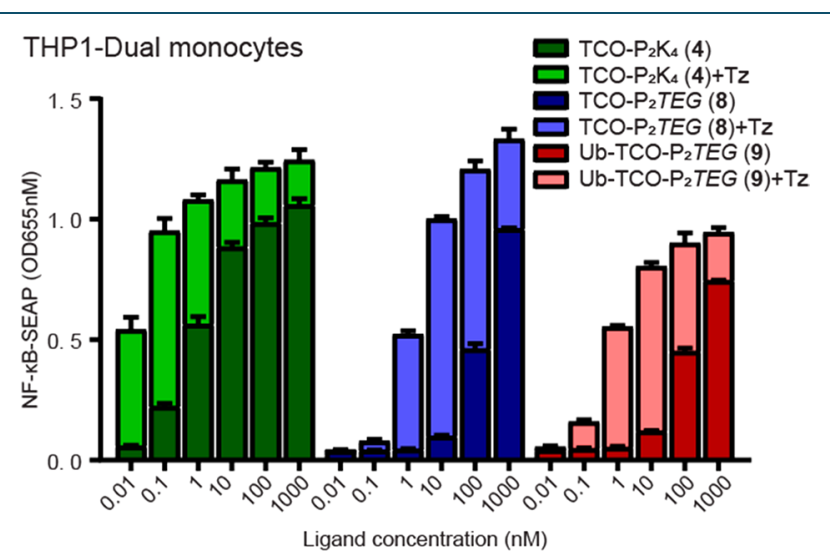

Figure 5. Substitution of the $\mathrm{K}_{4}$-tail with triethylene glycol (TEG) substantially reduces basal activity of the caged TLR2 ligands in human cells, which is not further improved by extensions on the TCO-cage. THP1-Dual reporter cells were treated as described in Figure $2 \mathrm{~A}$ and, after $24 \mathrm{~h}$, the level of SEAP in the culture supernatant was determined as a measure of NF- $\kappa \mathrm{B}$ activity. Data are representative of three independent experiments (whereof twice including compound 9).

similar results (Figure S3A) and, therefore, only fraction A is used in Figure 5. The activity of both compounds 8 and 9 was induced by $\mathrm{Tz}$, resulting in robust expression of the NF- $\kappa \mathrm{B}$ SEAP reporter (Figure 5). Generally, higher doses of the $\mathrm{P}_{2}$ TEG-derived compounds were required for eliciting similar levels of NF- $\kappa \mathrm{B}$ activity. This was true both for the deprotected (Figure 5) and free, noncapped ligands (Figure S3B). The UbTCO-modified 9 displayed much lower residual activity compared to 4 .

Surprisingly, 8 also induced comparably low levels of basal activity, despite the absence of a bulky TCO-substituent, yielding a good signal-to-noise ratio of NF- $\kappa \mathrm{B}$ activity in the $1-10 \mathrm{nM}$ concentration range (Figure 5). This suggests that the nonextended TCO sufficed to prevent receptor dimerization and that the $\mathrm{K}_{4}$-tail of compound 4 was the source of the 
background activity. The reason for this remains unelucidated. The $\mathrm{K}_{4}$-tail, but not the TEG, might provide modest opportunistic interactions that stabilize the TLR2/TLR6 receptor dimer, ${ }^{39}$ counteracting the protecting capacity of the TCO-cage. Nevertheless, 8 clearly is the preferred ligand for conditionally controlling human TLR2 signaling. Taken together, our data reveal that TCO- $\mathrm{P}_{2} \mathrm{TEG}(\mathbf{8})$ is a more suitable caged ligand for controlling human TLR2 activation than $\mathrm{TCO}-\mathrm{P}_{2} \mathrm{~K}_{4}(4)$, because it exerts potent inflammatory activity when deprotected without eliciting residual activity when protected.

Reaction Rate of Click-to-Release Uncaging Does Not Delay Immune Activation. Thus far, we have mainly focused on the efficacy of TCO as a chemical cage in terms of reducing and restoring signaling amplitude rather than the timing of receptor activation upon adding Tz. For precise temporal control of TLR2 activation, the uncaging reaction should occur rapidly, without causing an observable delay in dimerization-induced signaling, providing a "switch-like" activation upon adding the uncaging trigger.

To measure the time needed to induce TLR2 signaling events upon uncaging the ligand, we surveyed TLR2-induced nuclear translocation of NF- $\kappa \mathrm{B}$ over time. To this end, we performed live-cell imaging experiments on human TLR2 ${ }^{+}$ MelJuso cells that stably express a fluorescently labeled subunit of NF- $\kappa \mathrm{B}$ (p65-RFP). As expected from the above results, treatment of cells with $10 \mathrm{nM}$ of 7 resulted in a time dependent nuclear accumulation of NF- $\kappa \mathrm{B}$, whereas treatment with 8 did not (Figure 6A). Addition of $\mathrm{Tz}$ and continued imaging of the cells treated with 8 now revealed nuclear translocation of NF- $\kappa \mathrm{B}$ (Figure $6 \mathrm{~A}$ ).

For quantification purposes, $t=0$ was defined as the time when either free ligand was added to cells or when the uncaging reagent $\mathrm{Tz}$ was added to cells that had been pretreated with caged compound $\mathbf{8}$. This resulted in a similar temporal pattern of NF- $\kappa \mathrm{B}$ translocation in both settings (Figure $6 \mathrm{~B}$ ). The levels of NF- $\kappa \mathrm{B}$ in individual nuclei were analyzed over time (supplementary video). Compared to cells stimulated with free ligand 7 , no delay was detected in the speed with which nuclear NF- $\kappa$ B starts to accumulate after triggering uncaging of $\mathbf{8}$ (Figure $6 \mathrm{C}$ ). These data suggest that elimination of the TCO-cage occurs negligibly fast in human cells. Typical ligation kinetics are on the order of $10^{3} \mathrm{M}^{-1}$ $\mathrm{s}^{-131,48}$ and consequent elimination can proceed with a reaction constant of $0.06 \mathrm{~s}^{-1}\left(t_{1 / 2}=13 \mathrm{~s}\right)$ at physiological $\mathrm{pH}^{31,32}$ It should be noted that full recovery of agonist is likely not required for the induction of TLR2 signaling, meaning that the kinetics of NF- $\kappa$ B nuclear translocation may not accurately reflect the true uncaging kinetics. Still, the data illustrate the power of the Tz-induced "click-to-release" reaction for triggering the near-instantaneous activation of TLR2 in cells pretreated with TCO-P 2 TEG, making this (un)caging strategy extremely powerful for precisely controlling TLR2 activation.

\section{CONCLUSIONS}

In conclusion, we have shown trans-cyclooctene to be a suitable cage for preventing receptor activation when conjugated to a synthetic TLR2/6 agonist. We demonstrated that the activity of TCO-caged $\mathrm{P}_{2} \mathrm{~K}_{4}$ could be restored upon adding a tetrazine as an external trigger, which deprotects the ligand through a C2R-reaction. The conditional induction of TLR2 responses was validated in various murine cell types,

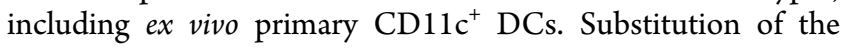

\section{A TLR2+ MelJuso p65-RFP cells}
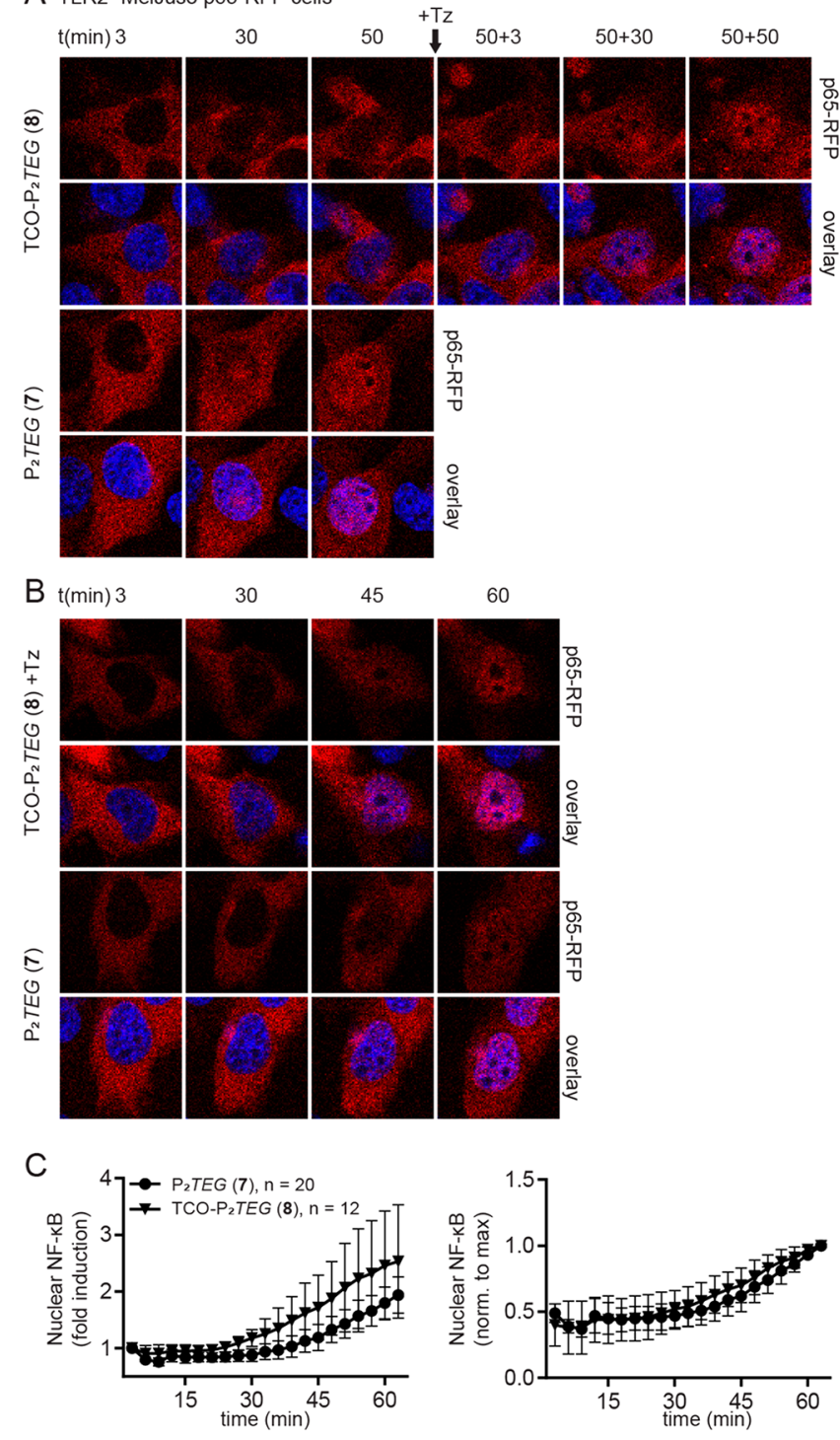

Figure 6. Tetrazine restores the activity of caged TLR2 ligands rapidly, without delaying $\mathrm{NF}-\kappa \mathrm{B}$ nuclear translocation kinetics. (AC) Live cell imaging of MelJuso TLR2-YFP p65-RFP cells. (A) Cells were simultaneously treated with indicated compounds and imaged for $50 \mathrm{~min}$. Subsequently, tetrazine $(\mathrm{Tz}, 10 \mu \mathrm{M})$ was added to the culture medium, and the same cells were imaged for an additional 50 min. (B,C) Cells were imaged after adding $\mathrm{Tz}(10 \mu \mathrm{M})$ to cells preincubated with $10 \mathrm{nM}$ TCO-P 2 TEG or after stimulation with $\mathrm{P}_{2}$ TEG. (B) Representative images of induced nuclear translocation of p65-RFP (in red) over time. (C) For individual cells, nuclear accumulation of NF- $\kappa \mathrm{B}$ was tracked over time: left, fold increase in nuclear p65-RFP fluorescence signal; right, normalized intensity of nuclear p65-RFP, relative to the maximum signal intensity observed. Quantification was performed on data from five independent experiments. (A,B) To indicate nuclei, overlays with Hoechst (in blue) are depicted.

terminal lysines of the prototypical agonist $\mathrm{P}_{2} \mathrm{~K}_{4}$ for a TEG yielded an easier to synthesize TCO-caged ligand with improved performance in human cells. Additionally, we showed that the kinetics of the uncaging reaction do not induce a detectable delay in TLR2 signaling, allowing temporal control over its activity. This methodology is not restricted to the TLR2/6 complex and may therefore also find its use for other pattern recognition receptors. Particularly suitable 
agonists for chemical caging have a low IC50 and are wellsoluble in aqueous solutions to ensure fast and complete deprotection via tetrazines. We foresee that the described IEDDA/pyridazine elimination strategy can form the basis of a chemical toolset for unraveling the complex spatial and temporal aspects of TLR signaling. Future work will aim at developing tetrazine derivatives to impose spatial control by directing the uncaging reagent to specific (sub)cellular sites. Controlling when and where TLRs are activated in cells will provide valuable insight into the complex spatiotemporal patterns of TLR regulation. A better understanding of the dynamic cellular processes that regulate TLR signaling may provide important therapeutic opportunities for modulating the (innate) immune responses, to combat infection or tumors, while preventing damage to the host due to destructive overinflammation.

\section{MATERIALS AND METHODS}

Detailed methods are described in the Supporting Information.

\section{ASSOCIATED CONTENT}

\section{(s) Supporting Information}

The Supporting Information is available free of charge at https://pubs.acs.org/doi/10.1021/acs.bioconjchem.0c00237.

Additional experimental data, spectra, and chromatograms; detailed methods for the chemical synthesis and biological assays; and supporting references (PDF)

Time-lapse video of p65-RFP levels in the nuclei of stimulated $\mathrm{TLR}^{+}$MJS cells used for quantification (Figure 6C) (AVI)

\section{AUTHOR INFORMATION}

\section{Corresponding Authors}

Sander I. van Kasteren - Department of Bio-Organic Synthesis, Leiden Institute of Chemistry, Leiden University, 2333 CC Leiden, Zuid-Holland, The Netherlands; (1) orcid.org/00000003-3733-818X; Email: s.i.van.kasteren@ chem.leidenuniv.nl

Maaike E. Ressing - Department of Cell and Chemical Biology, Leiden University Medical Center, 2333 ZC Leiden, ZuidHolland, The Netherlands; Email: m.e.ressing@lumc.nl

\section{Authors}

Michel J. van de Graaff - Department of Bio-Organic Synthesis, Leiden Institute of Chemistry, Leiden University, 2333 CC Leiden, Zuid-Holland, The Netherlands

Timo Oosenbrug - Department of Cell and Chemical Biology, Leiden University Medical Center, 2333 ZC Leiden, ZuidHolland, The Netherlands; (1) orcid.org/0000-0002-75330595

Mikkel H. S. Marqvorsen - Department of Bio-Organic Synthesis, Leiden Institute of Chemistry, Leiden University, 2333 CC Leiden, Zuid-Holland, The Netherlands

Clarissa R. Nascimento - INEM, INSERM, Unite 1151CNRS UMR 8253, Universite de Paris, 75015 Paris, France

Mark A. R. de Geus - Department of Bio-Organic Synthesis, Leiden Institute of Chemistry, Leiden University, 2333 CC Leiden, Zuid-Holland, The Netherlands; 이이.org/00000002-1639-5890

Bénédicte Manoury - INEM, INSERM, Unité 1151-CNRS UMR 8253, Universite de Paris, 75015 Paris, France

Complete contact information is available at: https://pubs.acs.org/10.1021/acs.bioconjchem.0c00237

\section{Author Contributions}

"M.J.v.d.G. and T.O. contributed equally. The manuscript was written through contributions of all authors. All authors have given approval to the final version of the manuscript.

\section{Funding}

M.J.v.d.G., M.A.R.d.G., and S.I.v.K. are funded by an ERC Starting Grant (Grant No. 639005). T.O. is funded by a grant from the NWO graduate program (Grant No. 022.006.010). M.H.S.M is funded by a Postdoc Abroad Fellowship of the Lundbeck Foundation. C.R.N. and B.M. are funded by INEM, INSERM, and PHC Van Gogh.

\section{Notes}

The authors declare no competing financial interest.

\section{ACKNOWLEDGMENTS}

We thank Prof. Aaron Esser-Kahn of the University of Chicago for providing the RAW Blue cells used in these experiments and the constructive discussion on the experiments in this manuscript. We thank Dr. Gerbrand J. van der Heden van Noort of the Leiden University Medical Center for his assistance in purifying compound $\mathbf{9}$ and consequent analysis hereof and Drs. Rob Hoeben and Sjaak Neefjes for valuable discussions. We would like to dedicate this paper to the memory of Dr. H. Ovaa.

\section{REFERENCES}

(1) Medzhitov, R. (2001) Toll-like receptors and innate immunity. Nat. Rev. Immunol. 1, 135-145.

(2) Pasare, C., and Medzhitov, R. (2004) Toll-like receptors: linking innate and adaptive immunity. Microbes Infect. 6, 1382-1387.

(3) Hennessy, E. J., Parker, A. E., and O’Neill, L. A. J. (2010) Targeting Toll-like receptors: emerging therapeutics? Nat. Rev. Drug Discovery 9, 293-307.

(4) Savva, A., and Roger, T. (2013) Targeting Toll-like receptors: promising therapeutic strategies for the management of sepsisassociated pathology and infectious diseases. Front. Immunol. 4, 387.

(5) Braunstein, M. J., Kucharczyk, J., and Adams, S. (2018) Targeting Toll-Like Receptors for Cancer Therapy. Target Oncol 13, $583-598$.

(6) Tsujimoto, H., Ono, S., Efron, P. A., Scumpia, P. O., Moldawer, L. L., and Mochizuki, H. (2010) Role of toll-like receptors in the development of sepsis. Shock 29, 315-321.

(7) Zuo, L., Lucas, K., Fortuna, C. A., Chuang, C. C., and Best, T. M. (2015) Molecular Regulation of Toll-like Receptors in Asthma and COPD. Front. Physiol. 6, 312.

(8) Lafyatis, R., and Marshak-Rothstein, A. (2007) Toll-like receptors and innate immune responses in systemic lupus erythematosus. Arthritis Res. Ther 9, 222.

(9) Tan, Y. H., and Kagan, J. C. (2017) Microbe-inducible trafficking pathways that control Toll-like receptor signaling. Traffic $18,6-17$.

(10) Park, B., Brinkmann, M. M., Spooner, E., Lee, C. C., Kim, Y. M., and Ploegh, H. L. (2008) Proteolytic cleavage in an endolysosomal compartment is required for activation of Toll-like receptor 9. Nat. Immunol. 9, 1407-1414.

(11) Maschalidi, S., Hassler, S., Blanc, F., Sepulveda, F. E., Tohme, M., Chignard, M., van Endert, P., Si-Tahar, M., Descamps, D., and Manoury, B. (2012) Asparagine Endopeptidase Controls AntiInfluenza Virus Immune Responses through TLR7 Activation. PLoS Pathog. 8, e1002841.

(12) Ewald, S. E., Engel, A., Lee, J., Wang, M. Q., Bogyo, M., and Barton, G. M. (2011) Nucleic acid recognition by Toll-like receptors is coupled to stepwise processing by cathepsins and asparagine endopeptidase. J. Exp. Med. 208, 643-651. 
(13) Garcia-Cattaneo, A., Gobert, F. X., Muller, M., Toscano, F., Flores, M., Lescure, A., Del Nery, E., and Benaroch, P. (2012) Cleavage of Toll-like receptor 3 by cathepsins $B$ and $H$ is essential for signaling. Proc. Natl. Acad. Sci. U. S. A. 109, 9053-9058.

(14) Lin, S. C., Lo, Y. C., and Wu, H. (2010) Helical assembly in the MyD88-IRAK4-IRAK2 complex in TLR/IL-1R signalling. Nature 465, 885-U2.

(15) Kagan, J. C., Su, T., Horng, T., Chow, A., Akira, S., and Medzhitov, R. (2008) TRAM couples endocytosis of Toll-like receptor 4 to the induction of interferon-beta. Nat. Immunol. 9, $361-368$.

(16) Yamamoto, M., Sato, S., Hemmi, H., Hoshino, K., Kaisho, T., Sanjo, H., Takeuchi, O., Sugiyama, M., Okabe, M., Takeda, K., et al. (2003) Role of adaptor TRIF in the MyD88-independent toll-like receptor signaling pathway. Science 301, 640-643.

(17) Barbalat, R., Lau, L., Locksley, R. M., and Barton, G. M. (2009) Toll-like receptor 2 on inflammatory monocytes induces type I interferon in response to viral but not bacterial ligands. Nat. Immunol. $10,1200-7$.

(18) Musilova, J., Mulcahy, M. E., Kuijk, M. M., McLoughlin, R. M., and Bowie, A. G. (2019) Toll-like receptor 2-dependent endosomal signaling by Staphylococcus aureus in monocytes induces type I interferon and promotes intracellular survival. J. Biol. Chem. 294, 17031-17042.

(19) Oosenbrug, T., van de Graaff, M. J., Ressing, M. E., and van Kasteren, S. I. (2017) Chemical Tools for Studying TLR Signaling Dynamics. Cell Chem. Biol. 24, 801-812.

(20) Marre, M. L., Petnicki-Ocwieja, T., DeFrancesco, A. S., Darcy, C. T., and $\mathrm{Hu}, \mathrm{L}$. T. (2010) Human Integrin alpha(3)beta(1) Regulates TLR2 Recognition of Lipopeptides from Endosomal Compartments. PLoS One 5, e12871.

(21) Stutts, L., and Esser-Kahn, A. P. (2015) A Light-Controlled TLR4 Agonist and Selectable Activation of Cell Subpopulations. ChemBioChem 16, 1744-1748.

(22) Ryu, K. A., Stutts, L., Tom, J. K., Mancini, R. J., and EsserKahn, A. P. (2014) Stimulation of Innate Immune Cells by LightActivated TLR7/8 Agonists. J. Am. Chem. Soc. 136, 10823-10825.

(23) Mancini, R. J., Stutts, L., Moore, T., and Esser-Kahn, A. P. (2015) Controlling the Origins of Inflammation with a Photoactive Lipopeptide Immunopotentiator. Angew. Chem., Int. Ed. 54, 59625965.

(24) Govan, J. M., Young, D. D., Lively, M. O., and Deiters, A. (2015) Optically triggered immune response through photocaged oligonucleotides. Tetrahedron Lett. 56, 3639-3642.

(25) Ryu, K. A., McGonnigal, B., Moore, T., Kargupta, T., Mancini, R. J., and Esser-Kahn, A. P. (2017) Light Guided In-vivo Activation of Innate Immune Cells with Photocaged TLR 2/6 Agonist. Sci. Rep. 7, 8074.

(26) Li, J., and Chen, P. R. (2016) Development and application of bond cleavage reactions in bioorthogonal chemistry. Nat. Chem. Biol. 12, 129-137.

(27) Tu, J. L., Xu, M. H., and Franzini, R. M. (2019) Dissociative Bioorthogonal Reactions. ChemBioChem 20, 1615-1627.

(28) Versteegen, R. M., Rossin, R., ten Hoeve, W., Janssen, H. M., and Robillard, M. S. (2013) Click to Release: Instantaneous Doxorubicin Elimination upon Tetrazine Ligation. Angew. Chem., Int. Ed. 52, 14112-14116.

(29) Blackman, M. L., Royzen, M., and Fox, J. M. (2008) Tetrazine ligation: fast bioconjugation based on inverse-electron-demand DielsAlder reactivity. J. Am. Chem. Soc. 130, 13518-9.

(30) Sarris, A. J. C., Hansen, T., de Geus, M. A. R., Maurits, E., Doelman, W., Overkleeft, H. S., Codee, J. D. C., Filippov, D. V., and van Kasteren, S. I. (2018) Fast and pH-Independent Elimination of trans-Cyclooctene by Using Aminoethyl-Functionalized Tetrazines. Chem. - Eur. J. 24, 18075-18081.

(31) de Geus, M. A. R., Maurits, E., Sarris, A. J. C., Hansen, T., Kloet, M. S., Kamphorst, K., Ten Hoeve, W., Robillard, M. S., Pannwitz, A., and Bonnet, S. A. (2020) Fluorogenic Bifunctional
Trans-cyclooctenes as Efficient Tools for Investigating Click-toRelease Kinetics. Chem.-Eur. J., DOI: 10.1002/chem.201905446.

(32) Carlson, J. C. T., Mikula, H., and Weissleder, R. (2018) Unraveling Tetrazine-Triggered Bioorthogonal Elimination Enables Chemical Tools for Ultrafast Release and Universal Cleavage. J. Am. Chem. Soc. 140, 3603-3612.

(33) Rossin, R., van Duijnhoven, S. M. J., ten Hoeve, W., Janssen, H. M., Kleijn, L. H. J., Hoeben, F. J. M., Versteegen, R. M., and Robillard, M. S. (2016) Triggered Drug Release from an Antibody-Drug Conjugate Using Fast "Click-to-Release" Chemistry in Mice. Bioconjugate Chem. 27, 1697-1706.

(34) Rossin, R., Versteegen, R. M., Wu, J., Khasanov, A., Wessels, H. J., Steenbergen, E. J., ten Hoeve, W., Janssen, H. M., van Onzen, A. H. A. M., Hudson, P. J., and Robillard, M. S. (2018) Chemically triggered drug release from an antibody-drug conjugate leads to potent antitumour activity in mice. Nat. Commun. 9, 1484.

(35) Li, J., Jia, S., and Chen, P. R. (2014) Diels-Alder reactiontriggered bioorthogonal protein decaging in living cells. Nat. Chem. Biol. 10, 1003-1005.

(36) Zhang, G., Li, J., Xie, R., Fan, X. Y., Liu, Y. J., Zheng, S. Q., Ge, Y., and Chen, P. R. (2016) Bioorthogonal Chemical Activation of Kinases in Living Systems. ACS Cent. Sci. 2, 325-331.

(37) Agustin, E., Asare Okai, P. N., Khan, I., Miller, M. R., Wang, R., Sheng, J., and Royzen, M. (2016) A fast click-slow release strategy towards the HPLC-free synthesis of RNA. Chem. Commun. 52, 14051408 .

(38) van der Gracht, A. M. F., de Geus, M. A. R., Camps, M. G. M., Ruckwardt, T. J., Sarris, A. J. C., Bremmers, J., Maurits, E., Pawlak, J. B., Posthoorn, M. M., Bonger, K. M., et al. (2018) Chemical Control over T-Cell Activation in Vivo Using Deprotection of transCyclooctene-Modified Epitopes. ACS Chem. Biol. 13, 1569-1576.

(39) Kang, J. Y., Nan, X., Jin, M. S., Youn, S. J., Ryu, Y. H., Mah, S., Han, S. H., Lee, H., Paik, S. G., and Lee, J. O. (2009) Recognition of Lipopeptide Patterns by Toll-like Receptor 2-Toll-like Receptor 6 Heterodimer. Immunity 31, 873-884.

(40) Botos, I., Segal, D. M., and Davies, D. R. (2011) The structural biology of Toll-like receptors. Structure 19, 447-59.

(41) Wu, W. Y., Li, R. T., Malladi, S. S., Warshakoon, H. J., Kimbrell, M. R., Amolins, M. W., Ukani, R., Datta, A., and David, S. A. (2010) Structure-Activity Relationships in Toll-like Receptor-2 Agonistic Diacylthioglycerol Lipopeptides. J. Med. Chem. 53, 3198-3213.

(42) Nordlander, J. E., Kotian, K. D., Raff, D. E., Njoroge, F. G., and Winemiller, J. J. (1984) Regiochemistry, Stereochemistry, and Mechanism of Addition of Trifluoroacetic-Acid to (Z)-Cyclooctene. J. Am. Chem. Soc. 106, 1427-1432.

(43) Omueti, K. O., Beyer, J. M., Johnson, C. M., Lyle, E. A., and Tapping, R. I. (2005) Domain exchange between human Toll-like receptors 1 and 6 reveals a region required for lipopeptide discrimination. J. Biol. Chem. 280, 36616-36625.

(44) Fan, X. Y., Ge, Y., Lin, F., Yang, Y., Zhang, G., Ngai, W. S. C., Lin, Z., Zheng, S. Q., Wang, J., Zhao, J. Y., et al. (2016)et al Optimized Tetrazine Derivatives for Rapid Bioorthogonal Decaging in Living Cells. Angew. Chem., Int. Ed. 55, 14046-14050.

(45) Jin, M. S., Kim, S. E., Heo, J. Y., Lee, M. E., Kim, H. M., Paik, S. G., Lee, H. Y., and Lee, J. O. (2007) Crystal structure of the TLR1TLR2 heterodimer induced by binding of a tri-acylated lipopeptide. Cell 130, 1071-1082.

(46) Grabiec, A., Meng, G. X., Fichte, S., Bessler, W., Wagner, H., and Kirschning, C. J. (2004) Human but not murine Toll-like receptor 2 discriminates between tri-palmitoylated and tri-lauroylated peptides. J. Biol. Chem. 279, 48004-48012.

(47) Wilkinson, B. L., Day, S., Malins, L. R., Apostolopoulos, V., and Payne, R. J. (2011) Self-Adjuvanting Multicomponent Cancer Vaccine Candidates Combining Per-Glycosylated MUC1 Glycopeptides and the Toll-like Receptor 2 Agonist Pam(3)CysSer. Angew. Chem., Int. Ed. 50, 1635-1639.

(48) Selvaraj, R., and Fox, J. M. (2013) trans-Cyclooctene - a stable, voracious dienophile for bioorthogonal labeling. Curr. Opin. Chem. Biol. 17, 753-760. 